Neutron Production Using a Pyroelectric Driven Target Coupled with a Gated Field Ionization Source

J. L. Ellsworth, V. Tang, S. Falabella, B. Naranjo, S. Putterman

August 3, 2012

22nd International Conference on the Application of Accelerators in Research and Industry Fort Worth, TX, United States August 5, 2012 through August 10, 2012 
This document was prepared as an account of work sponsored by an agency of the United States government. Neither the United States government nor Lawrence Livermore National Security, LLC, nor any of their employees makes any warranty, expressed or implied, or assumes any legal liability or responsibility for the accuracy, completeness, or usefulness of any information, apparatus, product, or process disclosed, or represents that its use would not infringe privately owned rights. Reference herein to any specific commercial product, process, or service by trade name, trademark, manufacturer, or otherwise does not necessarily constitute or imply its endorsement, recommendation, or favoring by the United States government or Lawrence Livermore National Security, LLC. The views and opinions of authors expressed herein do not necessarily state or reflect those of the United States government or Lawrence Livermore National Security, LLC, and shall not be used for advertising or product endorsement purposes. 


\title{
Neutron Production Using a Pyroelectric Driven Target Coupled with a Gated Field Ionization Source
}

\author{
J. L. Ellsworth ${ }^{\mathrm{a}}$ and V. Tang ${ }^{\mathrm{a}}$ and S. Falabella ${ }^{\mathrm{a}}$ and B. Naranjo ${ }^{\mathrm{b}}$ and S. Putterman ${ }^{\mathrm{b}}$ \\ ${ }^{a}$ Lawrence Livermore National Laboratory, 7000 East Ave., Livermore, CA 94550 \\ ${ }^{b}$ University of California Los Angeles, 405 Hilgard Ave., Los Angeles, CA 90095
}

\begin{abstract}
A palm sized, portable neutron source would be useful for widespread implementation of detection systems for shielded, special nuclear material. We present progress towards the development of the components for an ultracompact neutron generator using a pulsed, meso-scale field ionization source, a deuterated (or tritiated) titanium target driven by a negative high voltage lithium tantalate crystal. Neutron production from integrated tests using an ion source with a single, biased tungsten tip and a $3 \times 1 \mathrm{~cm}$, vacuum insulated crystal with a plastic deuterated target are presented. Component testing of the ion source with a single tip produces up to $3 \mathrm{nA}$ of current. Dielectric insulation of the lithium tantalate crystals appears to reduce flashover, which should improve the robustness. The field emission losses from a 3 $\mathrm{cm}$ diameter crystal with a plastic target and $6 \mathrm{~cm}$ diameter crystal with a metal target are compared.
\end{abstract}

Keywords: Neutron source, pyroelectric crystal, field ionization

PACS: $29.25 . \mathrm{Dz}, 29.25 . \mathrm{Ni}$

\section{INTRODUCTION}

A light-weight, ultra-compact, pulsed neutron source would be useful for detection of special nuclear materials via active interrogation [1]. Other applications include identification of organic explosives [2] and gas and oil well logging [3]. An accelerator based neutron source needs an ion source, a target, and HV insulation. The design we are pursuing uses a pyroelectric crystal to charge a tritiated titanium target to negative high voltage. The crystal charges up when heated so it can be powered by chemical sources rather than batteries, making it a lightweight option. Ions are created by a gated field ionization source constructed from sharp tungsten tips biased to positive high voltage and accelerated towards the target by the crystal's negative HV. Production of $10^{6} \mathrm{n} / \mathrm{s}$ using a tritiated target (or $10^{4} \mathrm{n} / \mathrm{s}$ using a deuterated target) at $-100 \mathrm{kV}$ requires $>50 \mathrm{nA}$ of $\mathrm{D}^{+}$ ion current.

The ion source is based on field ionization [4]. In the presence of the strong electric field created by applying a positive high voltage bias to a sharp metal tip, electrons are able to tunnel from the deuterium to the tip, creating a current of $\mathrm{D}^{+}$and/or $\mathrm{D}_{2}{ }^{+}$. Field strengths of $20-30 \mathrm{~V} / \mathrm{nm}$ are required for beams that have significant fractions of $\mathrm{D}^{+}$[5]. Gating the tip by surrounding it with a ground plane provides additional field enhancement.

In section 2, we describe a field ionization source that uses an array of commercially available, sharp tungsten tips mounted on a printed circuit board (PCB). The PCB has an embedded ground plane that gates the tips and current limiting resistors that prevent sustained discharges between the tips and ground. The tips can be biased positive to $10 \mathrm{~s}$ of $\mathrm{kV}$. Biasing the tip enhances the field so that the tip to crystal distance can be increased. This reduced arcing from the ion source to the crystal. Biasing the also allows for pulsed operation of the ion source by turning the bias on and off. An array of tips should multiply the current as long as the tips do not shield one another [6]. When a single tip is used, this ion source produced $\sim 3 \mathrm{nA}$ of current with a $+20 \mathrm{kV}$ bias.

Gillich [7] reports calculations showing that plastic targets have higher theoretical neutron yield than metal targets, but that plastic targets degrade under the experimental condition of pyrofusion. Deuterated titanium targets loaded 2:1 D:Ti (theoretically, the maximum limit) had the next highest theoretical yield. We are producing fully-loaded $\mathrm{TiD}_{2}$ targets $\left(\mathrm{Ti}: \mathrm{D}_{2}>\right.$ 1.7) and are setting up to produce tritium-loaded targets in the near future. We are using these targets with the $6 \mathrm{~cm}$ crystals in our experiments.

Lithium tantalate is a pyroelectric crystal. The surface of the crystal charges up when the crystal is thermal cycled. Z-cut $\mathrm{LiTaO}_{3}$ crystals with diameters 
as large as $3 \mathrm{~cm}$ have been successfully used in pyrofusion experiments and voltages greater than 100 $\mathrm{keV}$ have been measured [8-10]. Arcing and crystal robustness are a concern when crystals are insulated only by vacuum $[9,10] . \quad \mathrm{LiTO}_{3}$ has a nominal pyroelectric coefficient of $\mathrm{K}=170-190 \mu \mathrm{C} / \mathrm{m}^{2}{ }^{\circ} \mathrm{C}[9,11]$ so larger diameter crystals should be capable of producing more charge when thermal cycled. For the experiments described in this paper, the $+z$ face of the crystal is grounded and the $-z$ face is coupled to the target, such that the target is charged to negative high voltage when the crystal is heated. The ion beam produced by the ion source will then be accelerated towards the target. Biasing the target to negative $\mathrm{HV}$ provides some focusing for the ion beam, but field emission losses from metal targets are a potential concern.

Neutron production from integrated tests using a single tungsten tip on the ion source and a $3 \mathrm{~cm}$ diameter $x 1 \mathrm{~cm}$ thick, vacuum insulated crystal with a plastic deuterated target are presented in section 3 . In section 4, we present a new design for crystal insulation using a dielectric fluid that we are using with a large $6 \mathrm{~cm}$ diameter crystal. The secondary electron losses from a vacuum insulated crystal with a plastic target are compared to the losses from a dielectrically insulated, large-diameter crystal with a metal target.

\section{FIELD IONIZATION SOURCE}

The ion source uses electrochemically etched, 100 nm tungsten tips attached to a PCB board that has a buried ground plane and current limiting resistors. The board can accommodate an array of up to 12 tips, arranged in a circle, or one tip placed at the center of the circular array. Electrostatic modeling predicts the maximum electric field of one tip biased to $15 \mathrm{kV}$ is > $30 \mathrm{~V} / \mathrm{nm}$, which is sufficient for a few $\mathrm{nA}$ of $\mathrm{D}^{+}$ current. The PCB is entirely in vacuum and the tips can be biased to up to $+20 \mathrm{kV}$. The $\mathrm{HV}$ bias for the tips enters the chamber through a high voltage feedthrough. Fig. 1 shows a schematic view of the ion source.

The ion source was tested with a single, $100 \mathrm{~nm}$ tungsten tip in the center position. The field ionization current was measured by a collector biased to $+20 \mathrm{~V}$ and placed behind a grounded mesh. The ion source was located $\sim 1 \mathrm{~cm}$ from the grounded mesh. This distance is large enough that no field enhancement is expected from the mesh. The vacuum chamber was filled with 2.25 mTorr of D2 gas and the tip was biased to first $+10 \mathrm{kV}$, then $+15 \mathrm{kV}$, then $+10 \mathrm{kV}$ again and expected from the mesh. The vacuum chamber was filled with 2.25 mTorr of D2 gas and the

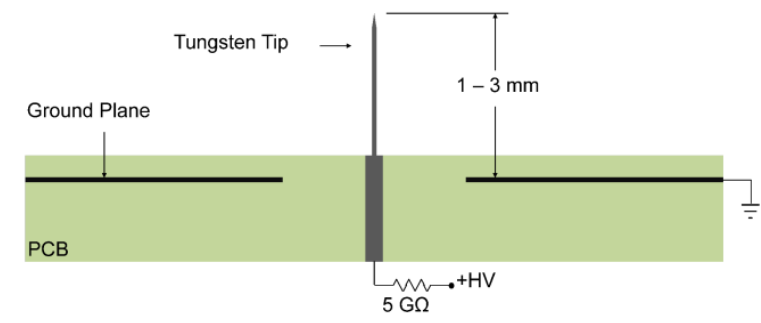

FIGURE 1. Schematic representation of a single tungsten tip on the ion source board including the embedded ground plane and the current protection resistor. The circuit board can accommodate arrays of up to twelve tips.

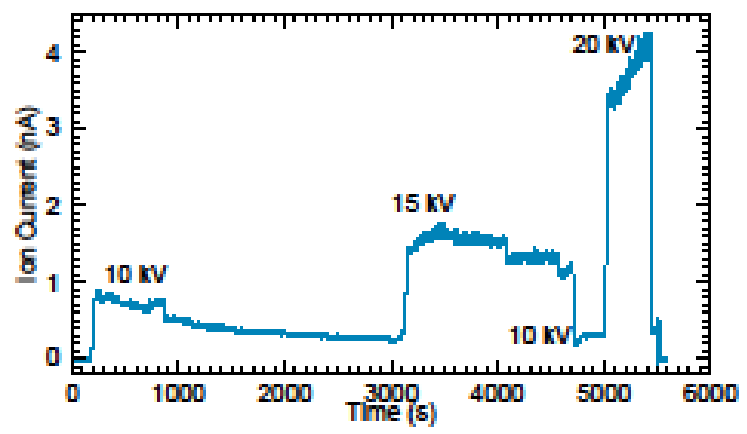

FIGURE 2. Field ionization current measured from a single biased, $100 \mathrm{~nm}$ tungsten tip in a chamber filled with $0.3 \mathrm{~Pa}$ of $\mathrm{D}_{2}$ gas.

tip was biased to first $+10 \mathrm{kV}$, then $+15 \mathrm{kV}$, then +10 $\mathrm{kV}$ again and $20 \mathrm{kV}$ (fig. 2). Increasing the bias voltage resulted in larger field emission current with a maximum of $\sim 3 \mathrm{nA}$ produced when the tip was biased to $+20 \mathrm{kV}$. When the tip was biased to $+10 \mathrm{kV}$, ion currents ranged from $0.3-0.8 \mathrm{nA}$. The composition of the ion beam was not measured.

\section{INTEGRATED TESTS WITH DECOUPLED NEGATIVE HIGH VOLTAGE CRYSTAL AND FIELD IONIZATION SOURCE LOADED WITH A SINGLE TUNGSTEN TIP}

Integrated testing of the ion source, target and crystal is necessary to make sure that this configuration is feasible. In our first round of testing, we used a $3 \mathrm{~cm}$ diameter, $3 \mathrm{~cm}$ thick, z-cut $\mathrm{LiTaO}_{3}$ crystal with a deuterated polystyrene $\left(\mathrm{CD}_{2}\right)$ target as a negative high voltage source and the vacuumfeedthrough field ionization source described in the previous section with a single, $100 \mathrm{~nm}$ tungsten tip. The $3 \times 3 \mathrm{~cm}$ crystal, temperature control system, and target are described in Ref. 10. From the component testing in the previous section, we expect $0.3-0.8 \mathrm{nA}$ of current from a single tip biased at $10 \mathrm{kV}$. This should produce a few hundred neutrons per second. 
The crystal was heated from $20 \mathrm{C}$ to $65 \mathrm{C}$ at 0.3 $\mathrm{C} / \mathrm{s}$ using a feedback-controlled thermoelectric cooler. The temperature of the crystal is measured by a thermocouple at the center of a thin copper disk that is glued to the base of the crystal. The gate valve to the vacuum pump was partially closed and deuterium gas was flowing through the chamber at a rate that held the vacuum pressure steady at $\sim 3$ mTorr. The tip on the ion source was biased using an external power supply after the crystal had charged up. The tip to target distance was $\sim 2 \mathrm{~cm}$.

Neutrons were measured by fourteen $\mathrm{He}^{3}$ tubes arrayed in a polyethylene moderator. The tubes detect neutrons by neutron capture. They are especially sensitive to thermal neutrons and essentially insensitive to gammas. This detector is a prototype version of the neutron detector used in the Ortec FMP3 portable neutron source identification system. The neutron detector was calibrated in the current experimental setup using a Cf- 252 source, which was placed as close to the target as possible. The measured Cf-252 efficiency was corrected by the MCNP calculated ratio of detection efficiency of D-D neutrons vs. Cf-252 neutrons. The resulting efficiency is $3 \pm 0.5 \%$ in the current setup. The background signal is $2-3 \mathrm{n} / \mathrm{s}$.

A sodium iodide detector measures the spectrum of bremsstrahlung emission that results from the stopping of secondary electrons emitted from the crystal. The endpoint of the bremsstrahlung spectrum is inferred to be the crystal voltage.

Arc detection is performed using a CCD camera. Single frames are collected every 2 seconds using a 2 second integration time. The images of the arcs are used to determine where arcing is occurring.

Fig. 3a shows the crystal temperature of the integrated test and fig. $3 \mathrm{~b}$ shows the neutron production taking the detector efficiency into account. A crystal voltage of $\sim-80 \mathrm{kV}$ after a $30 \mathrm{C}$ ramp is inferred from bremsstrahlung spectrum. For an $80 \mathrm{kV}$ potential and an ion beam with $<1 \mathrm{nA}$, we expected a neutron rate on the order of $10^{2} \mathrm{n} / \mathrm{s}$. The average neutron rate over the $106 \mathrm{~s}$ run is $1.6 \times 10^{2} \mathrm{n} / \mathrm{s}$. The peak neutron rate is higher. We suspect that there were some super-sharp areas of the tip that resulted in bursts of current $>1 \mathrm{nA}$. These super-sharp areas then burned off the tip.

Arc detection reveals that flashovers from the negative high voltage face of the crystal to the ground face are a concern, as expected from previous experiments $[9,10$,$] . We have chosen to pursue$ insulating the sides of a the crystal with a dielectric material to reduce the frequency of arcing from the
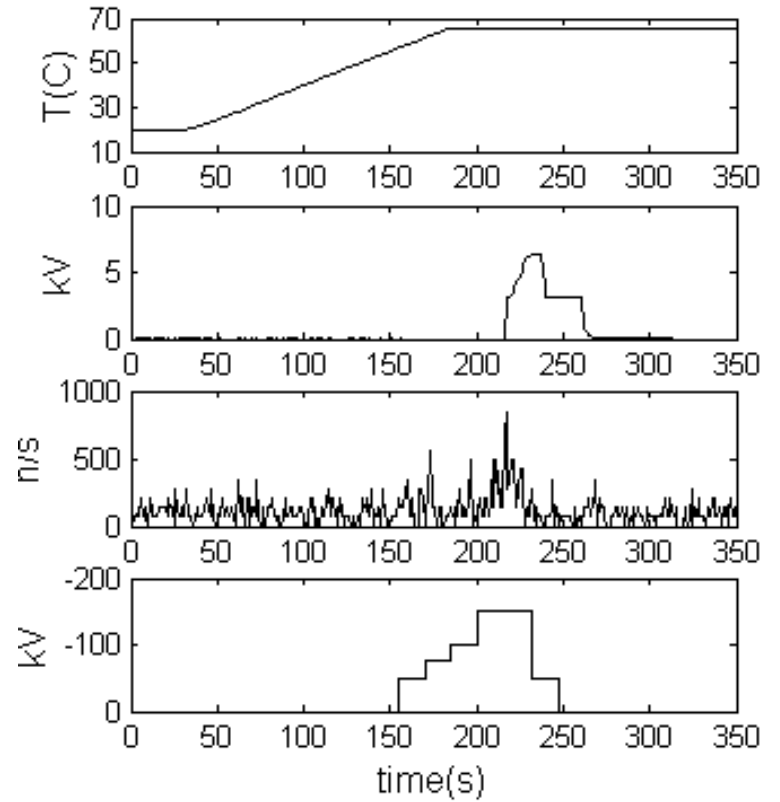

FIGURE 3. Results from an integrated test using a $3 \times 3 \mathrm{~cm}$ diameter crystal with plastic target and a single tungsten tip in the ion source. a) Measured crystal temperature. b) Ion source bias voltage. c) Neutron production averaged over 1 $\mathrm{s}$ time intervals, taking into account the detector efficiency. d) Crystal voltage measured from Bremsstrahlung emission.

negative high voltage face of the crystal to the ground plane.

\section{TESTS OF 6 CM NEGATIVE HIGH VOLTAGE CRYSTAL WITH METAL TARGET}

The $6 \mathrm{~cm}$ crystal assembly has been designed to provide improved insulation on the sides of the crystal by surrounding the sides of the crystal with a dielectric. In addition, the assembly clamps the metal target to the crystal without using an adhesive. This is advantageous for prototyping. O-ring seals isolate the dielectric from the vacuum. One of these o-ring seals is positioned at the edge of the crystal so that the oring acts as both a seal and a corona ring to prevent secondary electron emission. Figure 4 shows the design of the assembly and a photograph. The Lexan cup that is used to clamp the target to the crystal and the crystal to the thermoelectric cooler also acts as a vacuum vessel when placed on a flange with an o-ring seal.

The dielectric insulation surrounding the $6 \mathrm{~cm}$ crystal did reduce the flashover from the high voltage surface of the crystal to the ground side. We found that a liquid insulator was necessary because of the self- 


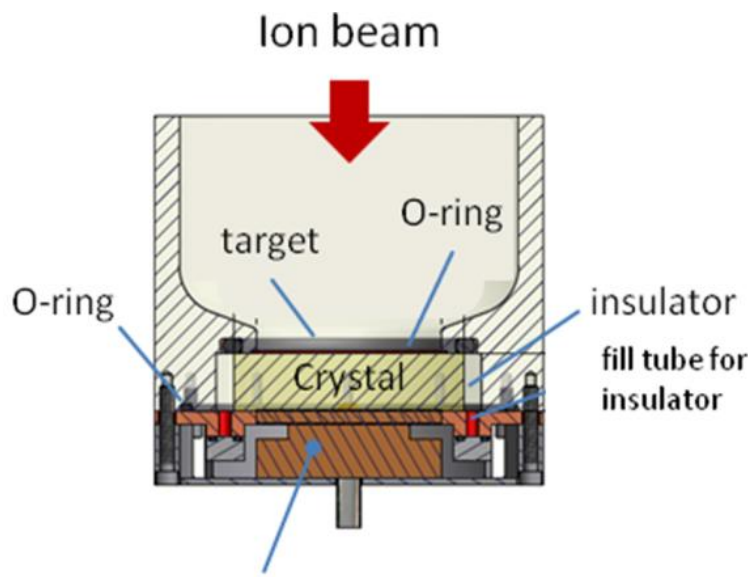

Heater/cooler

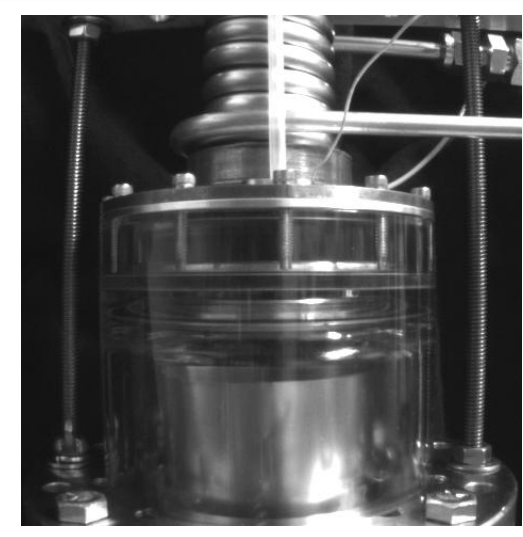

FIGURE 4. a) Drawing of the $6 \mathrm{~cm}$ crystal assembly. B) Photograph of the assembly as tested with the Faraday cup. The Lexan cup is 4" in diameter.

healing properties. These tests were performed using FC-43 as the dielectric. The design shown in fig. 4 required the top of the crystal to be metal plated and this plating was the only material holding vacuum if the crystal was damaged. The improved design used in the test we show had a metal plate with a curved edge between the crystal and the target so that the vacuum would not be ruined if the crystal was damaged. A thin piece of aluminum foil was used in between the crystal and the metal plate to improve the electrical contact. This eliminateed the need for plating the crystal.

The crystals were tested using a Faraday cup to measure secondary electron emission. The voltage on the crystal was inferred from bremsstrahlung emission measured by a sodium iodide detector. The $3 \times 3 \mathrm{~cm}$ crystal was measured with a hemispherical Faraday cup that extended all the way to the plane of the crystal for maximum charge collection. The $6 \times 1.5 \mathrm{~cm}$ crystal with metal target was measured with a cylindrical Faraday cup. (A hemispherical cup could not be used because of space constraints.) The top of this Faraday cup was placed $1 \mathrm{~cm}$ away from the target. We know
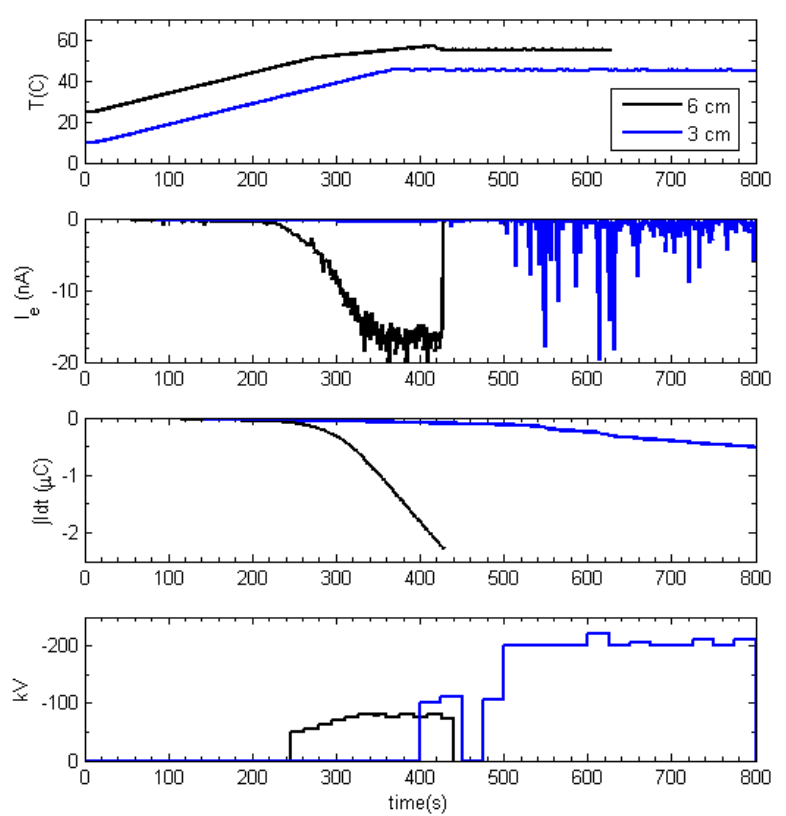

FIGURE 5. Measurements of field emission from a) $3 \times 3 \mathrm{~cm}$ crystal with plastic target and b) $6 \times 1.5 \mathrm{~cm}$ crystal with metal target show the crystal temperature, current measured by the Faraday cup, integrated charge, and crystal voltage measured from the endpoint of the Bremsstrahlung spectrum.

from experiments with unbiased probe tips that $1 \mathrm{~cm}$ is safe separation distance to avoid field ionization from the Faraday cup to the target.

Figure 5 compares the voltage and field emission loss measured from a $3 \times 3 \mathrm{~cm}$ crystal with a plastic target heated by $35 \mathrm{C}$ and a $6 \times 1.5 \mathrm{~cm}$ crystal with a metal target heated by $30 \mathrm{C}$. Using the given thermal cycles and $\mathrm{K}=170 \mu \mathrm{C} / \mathrm{m}^{2}{ }^{\circ} \mathrm{C}$ [9], the $3 \mathrm{~cm}$ diameter crystal has a maximum of $4.2 \mu \mathrm{C}$ and the $6 \mathrm{~cm}$ crystal has a maximum of $14.4 \mu \mathrm{C}$. The maximum charge available depends only on the surface area of the crystal [11], but the maximum voltage increases linearly with crystal thickness [12]. The $3 \times 3 \mathrm{~cm}$ assembly charges up to nearly $-200 \mathrm{kV}$ and the integrated charge loss is $.5 \mu \mathrm{C}$, or $12 \%$ of the maximum. The larger crystal with the metal target has an integrated charge loss of $2.3 \mu \mathrm{C}$ or $16 \%$ of the Other measurements of the voltage holding of the $6 \mathrm{~cm}$ crystal assembly, when a Faraday cup was not in place, have measured voltages exceeding $-100 \mathrm{kV}$.

\section{CONCLUSIONS}

Neutrons were produced in the decoupled configuration using a $3 \mathrm{~cm}$ diameter lithium tantalate crystal coated with a deuterated polystyrene target and a single, $100 \mathrm{~nm}$, gated tungsten tip biased to $+10 \mathrm{kV}$. Results of component testing for a gated ion source 
that used an array of biased tungsten tips were shown. A current of $\sim 3 \mathrm{nA}$ was measured from a single, 100 $\mathrm{nm}$ tungsten tip biased at $+20 \mathrm{kV}$. Large diameter $(6$ $\mathrm{cm}$ ), dielectrically insulated crystals were tested with metal targets. Initial testing shows that the dielectric insulation does reduce flashover. The fractional charge loss from the large diameter crystal with a metal target was comparable to the loss from a vacuum insulated, 3 cm crystal with a plastic target.

Future work includes further testing of the ion source with an array of tips as well as additional tests of the robustness of $6 \mathrm{~cm}$ diameter crystals. Integrated tests of the ion source with fully deuterated titanium targets and $6 \mathrm{~cm}$ crystals are also planned.

\section{ACKNOWLEDGMENTS}

The authors would like to thank Phil Kerr for assistance with the calibration of the neutron backpack diagnostic. This work was performed under the auspices of the U.S. Department of Energy by Lawrence Livermore National Laboratory under Contract DE-AC52-07NA27344 and supported by the U.S. Department of Energy NA-22 Office of Nonproliferation Research and Development under the Special Nuclear Materials Movement Detection portfolio.

\section{REFERENCES}

1. V. Tang and J. Morse and G. Meyer and S. Falabella and G. Guethlein and P. Kerr and H. G. Park and B. Rusnak and S. Sampayan and G. Schmid and C. Spadaccini and L. Wang. AIP Conference Proceedings 1099, 870 (2009).

2. P. C. Womble and F. J. Schultz and G. Vourvopoulos. Nucl. Instrum. Methods Physics Res. B, 99 757-760 (1999).

3. Committee on Radiation Source Use and Replacement, National Research Council. Radiation Source Use and Replacement, The National Academies Press, Washington, D.C. (2008).

4. R. Gomer, Surf. Sci., 299129 (1994).

5. T. C. Clements and E. W. Müller. J. Chem. Phys., 37 2684 (1962).

6. B. Bargsten Johnson and P.R. Schwoebel and C.E. Holland and P.J. Resnick and K.L. Hertz and D.L. Chichester . Nucl. Instrum. Methods Physics Res. A, 663 64-74 (2012).

7. D.J. Gillich and A. Kovanen and Y. Danon. J. Nucl. Mat., 405 181-185 (2010).

8. B. Naranjo and J. K. Gimzewski and S. Putterman. Nature, 434 1115-1117 (2005)

9. V. Tang and G. Meyer and S. Falabella and G. Guethlein and S. Sampayan and P. Kerr and B. Rusnak and J. D. Morse. J. Appl. Phys. 105026103 (2009).
10. D. J. Gillich and R. Teki and T. Z. Fullem and A. Kovanen and E. Blain and D. B. Chrisey and T. M Lu and Y. Danon. Nano Today, 4 227-234 (2009).

11. A. M. Glass. Phys. Rev., 172564 (1968).

12. J. Geuther, Y. Danon, F. Saglime, B. Sones, "Electron Acceleration for $\mathrm{X}$-ray Production Using Paired Pyroelectric Crystals", abstracts of the sixth International Meeting on Nuclear Applications of Accelerator Technology, AccApp'03, p. 591, San Diego, June 1-5, (2003). 